\title{
Organizational Leadership and Adaptive Reserve in Blood Pressure Control: The Heart Health NOW Study
}

Kamal H. Henderson, $M D^{1,2}$

Darren A. DeWalt, MD, MPH $H^{3,4}$

Jacquie Halladay, $M D, M P H^{2,4}$

Bryan J. Weiner, $\mathrm{PbD}^{5}$

Jung I. Kim, $P b D^{6}$

Jason Fine, $S_{c} D^{6}$

Samuel Cykert, $M D^{3,4}$

'Division of Cardiology, School of Medicine, University of North Carolina, Chapel Hill, North Carolina

${ }^{2}$ Division of Family Medicine, School of Medicine, University of North Carolina, Chapel Hill, North Carolina

${ }^{3}$ Division of General Medicine and Clinical Epidemiology, School of Medicine, University of North Carolina, Chapel Hill, North Carolina

${ }^{4}$ Cecil G. Sheps Center for Health Services Research, University of North Carolina, Chapel Hill, North Carolina

${ }^{5}$ Departments of Global Health and Biostatistics, University of Washington, Seattle, Washington

${ }^{6}$ UNC Gillings School of Global Public Health, University of North Carolina, Chapel Hill, North Carolina

Conflicts of interest: authors report none.

\section{CORRESPONDING AUTHOR}

Kamal H. Henderson, MD Division of Cardiology

The University of North Carolina at Chapel Hill

6th Floor, Burnett-Womack Building 160 Dental Circle

Chapel Hill, NC 27599-7075

kamal.henderson@unchealth.unc.edu

\begin{abstract}
PURPOSE Our purpose was to assess whether a practice's adaptive reserve and high leadership capability in quality improvement are associated with population blood pressure control.
\end{abstract}

METHODS We divided practices into quartiles of blood pressure control performance and considered the top quartile as the benchmark for comparison. Using abstracted clinical data from electronic health records, we performed a crosssectional study to assess the association of top quartile hypertension control and (1) the baseline practice adaptive reserve (PAR) scores and (2) baseline practice leadership scores, using modified Poisson regression models adjusting for practice-level characteristics.

RESULTS Among 181 practices, 46 were in the top quartile, which averaged 68\% or better blood pressure control. Practices with higher PAR scores compared with lower PAR scores were not more likely to reside in the top quartile of performance (prevalence ratio $[\mathrm{PR}]=1.92$ for highest quartile; $95 \% \mathrm{Cl}, 0.9-4.1$ ). Similarly, high quality improvement leadership capability compared with lower capability did not predict better blood pressure control performance (PR $=0.94$; $95 \% \mathrm{Cl}, 0.57-1.56)$. Practices with higher proportions of commercially insured patients were more likely than practices with lower proportions of commercially insured patients to have top quartile performance (37\% vs $26 \%, P=.002)$, whereas lower proportions of the uninsured $(8 \%$ vs $14 \%, P=.055)$ were associated with better performance.

CONCLUSIONS Our findings show that adaptive reserve and leadership capability in quality improvement implementation are not statistically associated with achieving top quartile practice-level hypertension control at baseline in the Heart Health NOW project. Our findings, however, may be limited by a lack of patientrelated factors and small sample size to preclude strong conclusions.

Ann Fam Med 2018;16(Suppl 1):S29-S34. https://doi.org/10.1370/afm.2210.

\section{INTRODUCTION}

$\mathrm{H}$ ealth care reform aimed at improving cost and quality of health care in the United States has encouraged many primary care practices to actively change how they deliver care. ${ }^{1,2}$

Implementing process improvement changes in the primary care setting is complex, with many factors necessary for successful change. Two intangible practice-level attributes have been shown in previous research to be particularly important: flexibility and resilience in time of change and primary care leadership that promotes inclusiveness and creates a culture of open communication. ${ }^{3-6}$ The practice adaptive reserve (PAR) scale is a commonly used assessment that quantifies successful work relationships that lead to flexibility and resilience within a practice. ${ }^{3}$ Conceptually, high PAR correlates with facilitative leadership and an organizational culture that values teamwork, improvisation, and sensemaking. ${ }^{7}$ Beyond facilitative leadership, the Key Drivers Implementation Scale (KDIS) is a newly developed measure that quantifies organizational leadership and integration of quality improvement in primary care practices. ${ }^{4,8}$ 
Both the PAR scale and KDIS are mostly used to evaluate and guide practice-level interventions aimed at changing cultural and contextual factors considered necessary to improve outcomes. ${ }^{4,8-12}$ The prevalence of a higher-level organizational culture and leadership, however, assessed by the PAR scale and KDIS at baseline, as well as their association with practice-level performance before implementing a quality improvement intervention, is not well understood. Do practices with leadership dedicated to continuous quality improvement and a practice-wide culture of adaptability associate with higher quality of care before actual facilitation has occurred? Research by Shing-Ping Tu et al did show a positive relationship between higher quartiles of baseline PAR scores in primary care clinics and better colorectal cancer screening, but it is unclear how these results translate to other baseline primary care practice performance measures related to chronic care. ${ }^{13}$

The objective of our study is to evaluate the association of PAR scores and leadership involvement in quality improvement with hypertension control before implementing multicomponent quality improvement interventions. Our hypothesis is that practices with higher levels of leadership involvement in quality improvement and clinical care teams with higher levels of adaptive reserve will be associated with better practice-level chronic care measures even before practice facilitation. For our analysis, we chose practice-level hypertension control performance as the sentinel measure of quality. We chose this outcome because it is an easily identifiable measure of cardiovascular care performance through electronic health records (EHRs) and a crucial measure for improved cardiovascular outcomes.

\section{METHODS}

Clinical data for our analysis were collected from the Heart Health NOW registry. Heart Health NOW is a stepped-wedge, stratified, cluster randomized trial studying the effect of providing primary care practices with an individual externally funded practice facilitator in addition to advanced informatics tools to support the provision of evidence-based cardiovascular disease care. ${ }^{14}$ Study procedures and methods were published previously. ${ }^{15}$ Two hundred twentyone small to medium-size primary care practices that manage adult patients enrolled and engaged in the study. Patient-level data for Heart Health NOW are extracted directly from practice EHRs and managed as a registry. All data collected within the registry are standardized and reported using established definitions. Before reporting, all data were normalized and underwent rigorous quality checks. For our analysis, we used a subset of practices for which EHR data are currently available. The Institutional Review Board at UNC Chapel Hill deemed the study exempt.

One month before practices were randomized to begin practice coaching, staff consisting of clinicians, nurses, and administrative personnel answered a Webbased survey questionnaire that included the PAR questionnaire and questions pertaining to practice characteristics. ${ }^{16}$ The PAR is an 18 -item self-administered questionnaire designed to measure the following domains: practice relationship infrastructure; alignment of practice management functions with practice operations and financial functions ${ }_{i}$ facilitative leadership ${ }_{i}$ teamwork ${ }_{i}$ positive work environment; and a culture of learning. Each question is assessed using a 5-point Likert scale. The PAR composite score was scaled from 0 to 1 for our analysis, with higher values representing greater agreement with the adaptive reserve domains assessed. Of the 989 practice members surveyed, 702 returned practice member questionnaires at baseline (71\% overall response rate). Each practice was assigned a mean adaptive reserve score based on the Web-based survey responses from each clinic site.

Practice facilitators assessed each practice for quality improvement leadership capability using KDIS at baseline. ${ }^{9,15}$ Created from the Chronic Care Model of chronic disease management, KDIS measures key factors necessary to implement successful practice-level process improvement changes. The KDIS evaluates practice-level quality improvement leadership capability on an ordinal rating from 0 to 3 , which indicates the following: score of 0 indicates little or no leadership support for quality improvement ${ }_{i} 1$ indicates that a leader is involved in quality improvement, but no organized improvement structure exists; 2 indicates leadership approaches improvement work as a project or task to be done; and 3 indicates that leadership recognizes quality improvement work as part of the daily routine and practice culture. ${ }^{4}$ The KDIS evaluation was conducted by practice coaches after each practice visit. Only the baseline visit is considered for this analysis.

Patients aged 18 to 85 years with an EHRdocumented diagnosis of hypertension who were seen within 12 months at the time of our data extraction were included in our analysis (from January 2015 to the date of the practice process improvement intervention). We excluded practices for which data extraction was incomplete at the time of our analysis.

Our outcome of interest was practice-level achievement in the top quartile of practices for rates of blood pressure control. The performance metric is the percentile for which adult patients aged 18 to 85 years diagnosed with hypertension and for whom the last clinic recorded blood pressure over a 12-month period was adequately controlled $(<140 / 90 \mathrm{~mm} \mathrm{Hg})$. 


\section{Table 2. Practice-Level Comparison of Top Quartile Hypertension Performance}

\begin{tabular}{|c|c|c|c|}
\hline \multirow[b]{2}{*}{$\begin{array}{l}\text { Practice-Level } \\
\text { Characteristic }\end{array}$} & \multicolumn{3}{|c|}{ Top Quartile Hypertension } \\
\hline & $\begin{array}{c}\text { Top Quartile } \\
\text { Achieved } \\
n=46\end{array}$ & $\begin{array}{c}\text { Top Quartile } \\
\text { Not Achieved } \\
n=135\end{array}$ & $\begin{array}{c}P \\
\text { Value }\end{array}$ \\
\hline Clinicians, mean No. (SD) & $5(5.4)$ & $5.6(4.8)$ & .596 \\
\hline Office staff, mean No. (SD) & $8.6(11.4)$ & $8.3(7.8)$ & .917 \\
\hline Physician owned, No. (\%) & $27(35)$ & $50(65)$ & .015 \\
\hline \multicolumn{4}{|l|}{ Payer mix } \\
\hline Medicare, \% (SD) & $28(19.2)$ & $29(14.8)$ & .776 \\
\hline Medicaid, \% (SD) & $15(11.6)$ & $17(11.7)$ & .477 \\
\hline Commercial insurance, \% (SD) & $37(15.9)$ & $26(18.3)$ & .002 \\
\hline Uninsured, \% (SD) & $8(9.5)$ & $14(14.7)$ & .055 \\
\hline \multicolumn{4}{|l|}{ Practice delivery model } \\
\hline PCMH, No. (\%) & $29(33)$ & $60(67)$ & .014 \\
\hline FQHC, No. (\%) & $5(15)$ & $29(85)$ & .179 \\
\hline \multicolumn{4}{|l|}{$\begin{array}{l}\text { Organizational quality improve- } \\
\text { ment quality }\end{array}$} \\
\hline PAR score, mean (SD) ${ }^{a}$ & $0.72(0.11)$ & $0.67(0.11)$ & .165 \\
\hline \multicolumn{4}{|l|}{ KDIS leadership score ${ }^{b, c}$} \\
\hline 0 & 7 & 9 & $\ldots$ \\
\hline 1 & 17 & 59 & $\ldots$ \\
\hline 2 & 14 & 42 & $\ldots$ \\
\hline 3 & 8 & 25 & $\ldots$ \\
\hline \multicolumn{4}{|c|}{$\begin{array}{l}\mathrm{FQHC}=\text { federally qualified health center; } \mathrm{KDIS}=\text { Key Driver Implementation Scale; } \mathrm{PAR}=\text { prac- } \\
\text { tice adaptive reserve; } \mathrm{PCMH}=\text { patient-centered medical home; } \mathrm{QI}=\text { quality improvement. }\end{array}$} \\
\hline \multicolumn{4}{|c|}{$\begin{array}{l}\text { a PAR scores are scaled from } 0 \text { to } 1 \text {, with } 1 \text { being a perfect score of agreement for organiza- } \\
\text { tional adaptiveness. }\end{array}$} \\
\hline \multicolumn{4}{|c|}{$\begin{array}{l}{ }^{\mathrm{b}} \mathrm{KDIS} \text { Leadership scores are scaled from } 0 \text { to } 3 \text {, with } 3 \text { being a perfect score that leadership } \\
\text { recognizes QI work as part of the daily routine and practice culture. }\end{array}$} \\
\hline \multicolumn{4}{|c|}{$\begin{array}{l}{ }^{c} P=.356 \text {, estimated from } \chi^{2} \text { analysis comparing ordinal leadership scores for all practices } \\
\text { achieving top quartile hypertension control vs not achieving top quartile hypertension control. }\end{array}$} \\
\hline
\end{tabular}

The prevalence of top quartile blood pressure control performance also did not differ by leadership score (Table 3 ).

Practice-level blood pressure control measure performance by organizational quality improvement characteristics are shown in Figure 1. Predicted performance was $4 \%$ higher for the higher PAR quartiles when compared ...with the lowest PAR quartile (middle 2 quartiles were combined), but was not statistically significant $(61 \%$ vs $57 \% ; P=.504)$. Again, differing leadership levels did not predict different blood pressure control performance.

\section{DISCUSSION}

Successful implementation of quality improvement strategies can be affected by both organizational contextual facilitators and barriers. There is a paucity of literature of how organizational contextual factors, such as adaptive reserve and quality improvement leadership capability, relate to blood pressure control performance in primary care clinics, particularly without ongoing formal quality improvement advice or externally driven interventions. In this cross-sectional study consisting of 181 practices providing primary care to adult patients throughout North Carolina, we explored the association of organizational

included in our analysis, 104,473 patients among practices met adequate hypertension control (61\%). Uninsured patients represented a small proportion of the payer mix among practices. At baseline, most practices reported a high degree of organizational adaptive reserve, whereas approximately one-half of the practices had high leadership scores.

Bivariate analysis of baseline practice-level characteristics and target hypertension performance can be seen in Table 2. There were no substantial differences in performance on the hypertension measure by number of clinicians or number of office staff. Practices in the top quartile had a statistically higher percentage of patients with commercial insurance at $37 \%$ vs $26 \%$ $(P=.002)$, and a lower percentage of uninsured patients at $8 \%$ vs $14 \%(P=.055)$.

Prevalence ratios for top quartile blood pressure control performance, both adjusted and unadjusted as assessed by PAR and KDIS Leadership, are shown in Table 3. We found a weak correlation with increasing interquartile PAR scores among practices and top quartile performance that was not statistically significant. quality improvement characteristics with target hypertension performance before implementing a quality improvement intervention. Our analysis has 2 relevant findings to be considered. First, our results suggest that such organizational characteristics as greater adaptive reserve and strong quality improvement leadership are not associated with a statistically significant better performance at baseline. Second, meeting specific practicelevel quality improvement performance targets can be influenced by the payer-mix distribution at a practice despite positive quality improvement characteristics before formal practice facilitation has occurred.

PAR and KDIS have mostly been used as part of ongoing evaluations of organizations to predict outcomes after a quality improvement implementation intervention. They are also used to inform the team supporting change in the practice..$^{8,11,13}$ Our study differs in using a cross-sectional assessment of adaptive reserve and quality improvement leadership to evaluate current hypertension care before practice facilitation. The lack of statistically significant difference in blood pressure control measure performance between 
low and higher PAR-scoring practices is contradictory to the findings of Shing-Ping Tu, et al, showing that higher quartiles of PAR scores were related to higher predicted mean colorectal cancer screening. One must consider, however, based on the degree of association between the PAR scores and the primary outcome of

\begin{tabular}{|c|c|c|c|c|}
\hline $\begin{array}{l}\text { Quality Improvement } \\
\text { Contextual Factors }\end{array}$ & PR (Cl) & $\begin{array}{c}P \\
\text { Value }\end{array}$ & $\begin{array}{l}\text { Adjusted } \\
\text { PR (Cl) }\end{array}$ & $\begin{array}{c}P \\
\text { Value }\end{array}$ \\
\hline \multicolumn{5}{|l|}{ Leadership ${ }^{b}$} \\
\hline Low leadership (score 0-1) & 1 [Referent] & & 1 [Referent] & \\
\hline High leadership (score 2-3) & $0.94(0.57-1.56)$ & .833 & $0.81(0.48-1.37)$ & .429 \\
\hline \multicolumn{5}{|l|}{ PAR scorec } \\
\hline 0.00 to $<0.66$ & 1 [Referent] & & 1 [Referent] & \\
\hline 0.65 to $<0.76$ & $1.47(0.71-3.03)$ & .297 & $1.25(0.52-2.99)$ & .611 \\
\hline 0.76 to 1.00 & $1.92(0.9-4.1)$ & .091 & $1.45(0.56-3.76)$ & .440 \\
\hline \multicolumn{5}{|l|}{ High leadership + PAR scores ${ }^{d}$} \\
\hline High leadership & $0.93(0.57-1.53)$ & .776 & & \\
\hline $\operatorname{PAR}(0.65$ to $<0.76)$ & $1.48(0.72-3.05)$ & .289 & & \\
\hline PAR (0.76 to 1.00$)$ & $1.92(0.9-4.09)$ & .089 & & \\
\hline
\end{tabular}

$\mathrm{PAR}=$ practice adaptive reserve; $\mathrm{PR}=$ prevalence ratio.

a Modified Poisson regression models adjusted for the following: proportion of commercially insured patients, practice designation as a patient-centered medical home, and clinician-owned practices

${ }^{b}$ PR: prevalence for top quartile hypertension control among practices with high leadership support for quality improvement implementation divided by the prevalence for top quartile hypertension control among practices with less leadership support for quality improvement implementation.

c PR: prevalence for achieving top quartile hypertension control among practices with higher quartiles of PAR divided by the prevalence for top quartile hypertension control among practices with the lowest quartile of PAR.

d PR reflects adjustments for both high leadership support for quality improvement implementation and higher quartiles of PAR when compared to the referent (low leadership support for quality improvement implementation and lowest quartile of PAR).

Figure 1. Predicted practice-level performance on blood pressure control measure stratified by organizational quality improvement characteristics.

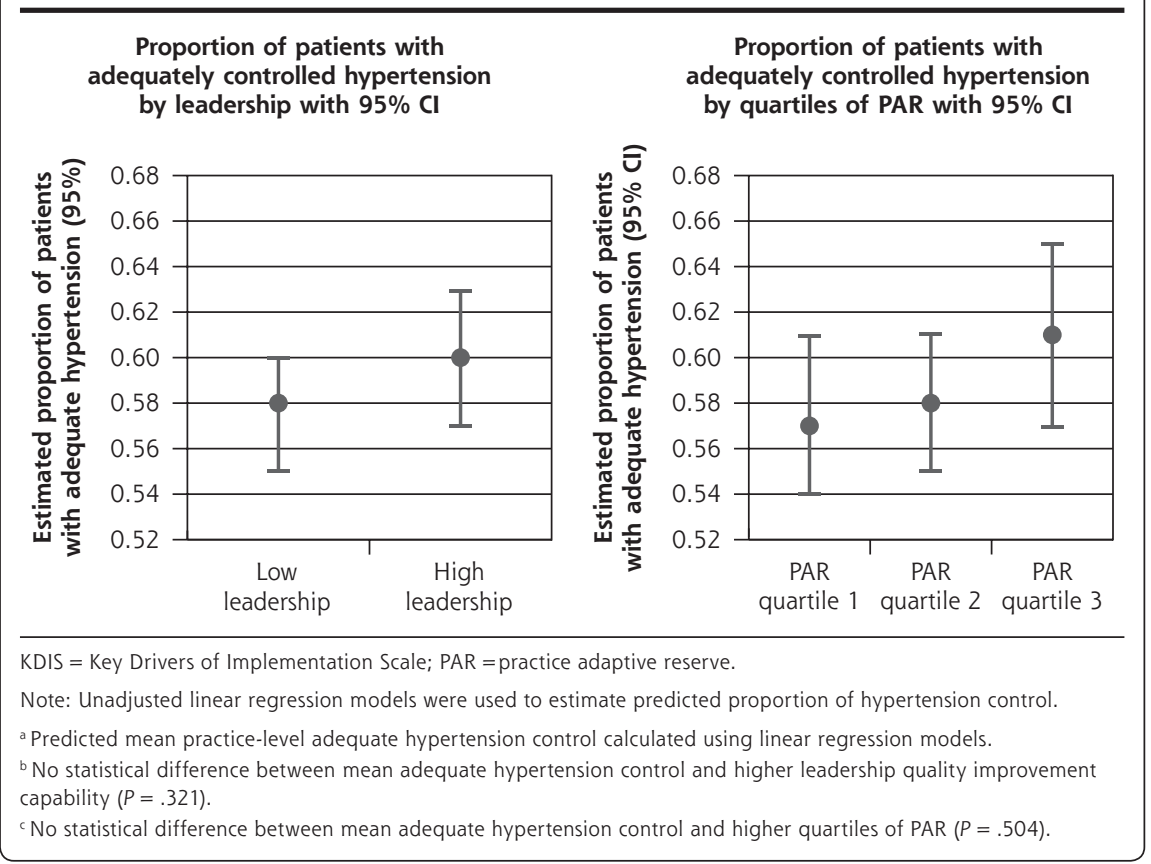

top quartile hypertension control, that a larger sample size could result in statistically significant findings. Post hoc power calculations were conducted using the estimated effect sizes for PAR scores and high leadership scores plus PAR scores on target hypertension in Table 3. To detect a prevalence ratio of roughly 1.5 based on our population of study sites using a Wald test at an $\alpha$ level of .05 based on the estimated regression parameter from the modified Poisson regression, more than 1,000 representative sites would be needed for $80 \%$ power. To similarly detect a prevalence ratio of roughly 1.9 , approximately 500 sites would be needed for $80 \%$ power. Thus, although the estimated trend in the effect sizes from lower to higher adaptive reserve is suggestive, much larger sample sizes would be needed to show the statistical significance of these small to moderate adaptive reserve effects.

Although our findings do not suggest a statistical association with our outcome of interest (blood pressure control), other unidentified factors outside these contextual factors are likely responsible for high baseline performance. Factors that affect population-level hypertension control, such as effective engagement and communication, the actual filling of multiple prescriptions, consistent adherence, and lifestyle counseling, are complex and not easily captured in EHR systems. Even if a practice has adaptive reserve and leadership capability, these characteristics may not be able overcome the disadvantage of no or underinsurance.

As with any study, there are several limitations that 
must be considered. Our analysis was performed using a subset of all the practices in Heart Health NOW. It is possible that this sample is biased compared with the total number of practices, limiting generalizability of our results. Also, we did not assess the impact of individual patient demographic and health characteristics because this analysis was at a practice level. Although this analysis was at a practice level, some clinically meaningful predictor variables may have interacted with organizational variables and changed our results, such as what clinicians considered to be adequate hypertension control for their patients and the comorbidities of the patients they treated. Also, only 46 practices were in the top quartile for hypertension control, and a small sample size may have limited the power of the Poisson regression models to estimate an association between top quartile hypertension control and our independent variables.

In conclusion, within a broad range of small, primary care practices, adaptive reserve and leadership capability in quality improvement implementation are not statistically associated with achieving top quartile practicelevel hypertension control at baseline in the Heart Health NOW project. Our findings, however, may be limited by a lack of patient-related factors and a small sample size to preclude strong conclusions. Practicelevel quality improvement assessments may have utility in understanding a practice's quality improvement culture before implementing practice-level redesign efforts but may not matter until appropriate facilitation and tools for system redesign are available, especially for measures related to chronic disease management. Adaptive reserve and quality improvement leadership may be predictors of capacity to improve once there is an applied intervention that takes the many complex components of chronic care into consideration.

To read or post commentaries in response to this article, see it online at http://www.AnnFamMed.org/content/16/Suppl_1/S29.

Key words: blood pressure control; quality improvement; primary health care; hypertension; leadership; population health; cardiovascular disease

Submitted June 19, 2017; submitted, revised, January 21, 2018; accepted January 24, 2018.

Funding support: Publication of this article was supported by the Agency for Healthcare Research and Quality (AHRQ) through contract No. HHSA290201200019l, and grant No. R18 HS023912.

Disclaimer: This work represents the opinions of the authors and should not be interpreted as official positions of the Agency for Healthcare Research and Quality or the US Department of Health and Human Services.

\section{References}

1. Centers for Medicare and Medicaid Services. Proposed Rule for Quality Payment Program Year 2. https://www.cms.gov/Medicare/ Quality-Initiatives-Patient-Assessment-Instruments/Value-BasedPrograms/MACRA-MIPS-and-APMs/Proposed-Rule-for-the-QualityPayment-Program-Year-Two-Slides.pdf.

2. Pizzo JJ, Pryor RW, York RW. Ready, Set, Go: Helping Physicians Move to Value as MACRA Begins. J Healthc Manag. 2016;61(6): 396-401.

3. Miller WL, Crabtree BF, Nutting PA, Stange KC, Jaén CR. Primary care practice development: a relationship-centered approach. Ann Fam Med. 2010;8 Suppl 1:S68-79; S92.

4. Donahue KE, Halladay JR, Wise A, et al. Facilitators of transforming primary care: a look under the hood at practice leadership. Ann Fam Med. 2013;11(Suppl 1):S27-S33.

5. Bowers KW, Robertson M, Parchman ML. How inclusive leadership can help your practice adapt to change. Fam Pract Manag. 2012; 19(1):8-11.

6. Nutting PA, Crabtree BF, Stewart EE, et al. Effect of facilitation on practice outcomes in the National Demonstration Project model of the patient-centered medical home. Ann Fam Med. 2010;8 Suppl 1:S33-44; 592.

7. Jordan Z. Magnet recognition and practice development: two journeys towards practice improvement in health care. Int J Nurs Pract. 2009;15(6):495-501.

8. Halladay JR, DeWalt DA, Wise A, et al. More extensive implementation of the chronic care model is associated with better lipid control in diabetes. J Am Board Fam Med. 2014;27(1):34-41.

9. Crabtree BF, Nutting PA, Miller WL, Stange KC, Stewart EE, Jaén CR. Summary of the National Demonstration Project and recommendations for the patient-centered medical home. Ann Fam Med. 2010;8 Suppl 1:S80-90; S92.

10. Cohen D, McDaniel RR Jr, Crabtree BF, et al. A practice change model for quality improvement in primary care practice. J Healthe Manag. 2004;49(3):155-168, discussion 169-170.

11. Solberg LI, Crain AL, Jaeckels N, et al. The DIAMOND initiative: implementing collaborative care for depression in 75 primary care clinics. Implement Sci. 2013;8:135.

12. Shea CM, Jacobs SR, Esserman DA, Bruce K, Weiner BJ. Organizational readiness for implementing change: a psychometric assessment of a new measure. Implement Sci. 2014;9:7.

13. Tu SP, Young VM, Coombs LJ, et al. Practice adaptive reserve and colorectal cancer screening best practices at community health center clinics in 7 states. Cancer. 2015;121(8):1241-1248.

14. Kohli $P$, Whelton SP, Hsu S, et al. Clinician's guide to the updated ABCs of cardiovascular disease prevention. J Am Heart Assoc. 2014; 3(5):e001098.

15. Weiner BJ, Pignone MP, DuBard CA, et al. Advancing heart health in North Carolina primary care: the Heart Health NOW study protocol. Implement Sci. 2015;10:160.

16. Jaén $C R$, Crabtree BF, Palmer RF, et al. Methods for evaluating practice change toward a patient-centered medical home. Ann Fam Med. 2010;8 Suppl 1:S9-20; S92.

17. Measure \#236 (NQF 0018): Controlling High Blood Pressure National Quality Strategy Domain: Effective Clinical Care. https: //www.qualityforum.org/Measuring_Performance/Measuring_ Performance.aspx. Published 2017.

18. Zou G. A modified Poisson regression approach to prospective studies with binary data. Am J Epidemiol. 2004;159(7):702-706. 